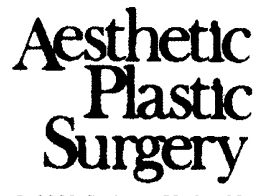

(c) 2003 Springer-Verlag New York Inc

\title{
Composite Skin Grafts for Basal Cell Carcinoma Defects of the Nose
}

\author{
Raffi Gurunluoglu, Maziar Shafighi, Alexander Gardetto, and Hildegunde Piza-Katzer \\ Innsbruck, Austria
}

\begin{abstract}
Basal cell carcinoma is the most frequent cutaneous cancer of the nose and is characterized by its local spreading and exceptionally rare tendency to metastasize. Since a significant advantage has been seen in surgery compared to other treatments, surgical excision ensuring the highest chance of cure is frequently employed. Excision defects of the nose may be covered with either local flap or a full-thickness skin graft. In resurfacing such defects following excision of basal cell carcinomas, we favor the technique of composite-skin grafting which involves the harvesting of composite-skin graft including the epidermis, dermis and superficial layers of subcutaneous tissue to obtain the required thickness in the recipient site. This technique was used for defects remaining after the excision of basal cell carcinomas in a series of 15 patients. The areas involved were lateral nasal region ( 5 cases), nasal tip (4 cases), dorsum ( 3 cases), alar lobule ( 2 cases), and soft triangle ( 1 case). The mean follow-up was 14.2 months. The color, texture and thickness of the composite-skin graft harvested from the preauricular site and the neck compare favorably with the skin of the nose region. Satisfactory results, both clinically and in patient appreciation, have been obtained in both the reconstruction site and the appearance of the donor site in all patients.
\end{abstract}

Key words: Basal cell carcinoma-Nose-Skin graft-Local flap

Basal cell carcinoma is by far the most common malignancy of whites, arising from cells of the basal layer of the epithelium or from the external root

Correspondence to Raffi Gurunluoglu, Department of Plastic and Reconstructive Surgery,, Leopold - Franzens University Clinic Innsbruck, Anichstrasse, 35, A - 6020 Innsbruck, Austria; email: rgurunluoglu@hotmail.com sheath of the hair follicle. The majority of basal cell carcinomas occur in fair-skinned individuals who have been exposed to the sun, x-irradiation, or ultraviolet light for prolonged periods. Head and neck are the most susceptible areas of the body for this type of skin lesion [1-3].

It would be difficult to describe a form of treatment that has not been used for basal cell carcinomas. To date, a variety of treatment methods depending on the type, size, site of basal cell carcinoma, age of the patient, patient's and surgeon's preference have been employed for basal cell carcinoma. Treatment planning assumes that achievement of local control is synonymous with cure. Some basal cell carcinomas are treated by curettage and desiccation or by simple excision as a fusiform ellipse and primary closure. The surgeon has a choice of closures, i.e., immediate versus delayed and primary closure versus reconstruction with local flap or skin graft depending on the pathology encountered and the availability of frozen section diagnosis. Other methods less widely used include irradiation, cryotherapy, and topical application of a chemical such as 5-FU [1],

Among various treatment modalities, surgical excision provides an immediate pathologic inventory and an index of the adequacy of excision. Excision with margins and closure with a local flap or a skin graft is mostly employed for large lesions. While providing the best color and texture match, problems associated with the use of local flaps are not uncommon. They include contour deformities and increased scarring due to multiple incisions which are often required in flap surgery. Moreover, formation of dog-ears requiring secondary operations is not infrequent. Our preference for coverage of defects after basal cell carcinoma ablation is to use a composite-skin graft preferably from the preauricular region or the neck. In this paper, we present our experience in a series of 15 patients who underwent basal cell carcinoma defect closure with composite- 

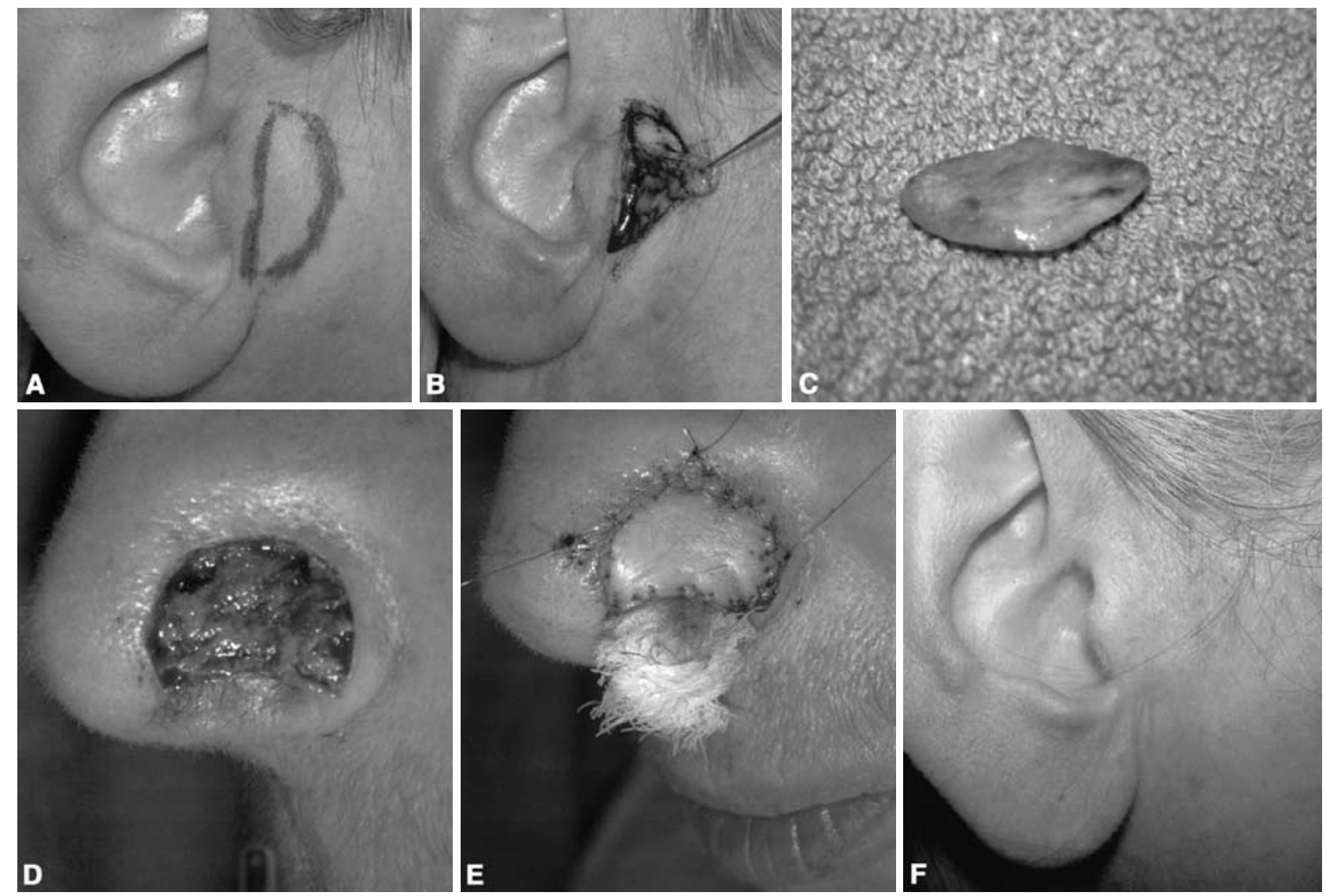

Fig. 1. Illustration of the composite-skin grafting technique in a 54-year-old female patient (Patient 1): (A) Close-up picture illustrating preoperative planning for harvesting the composite-skin graft from preauricular site, (B) the level of subcutaneous dissection for obtaining a composite-skin graft that includes a superficial layer of subcutaneous tissue, (C) the composite-skin graft, (D) defect at the left alar region following excision of a basal cell carcinoma, (E) closure of the defect with compositeskin graft, (F) appearance of the preauricular graft donor site at 11-month follow-up.

skin graft over the past 5 years. In addition, several pitfalls have been highlighted to obtain the best possible aesthetic outcome.

\section{Surgical Technique}

All patients have been operated on under field block or regional anesthesia. The basal cell carcinoma was excised with $2-3 \mathrm{~mm}$ healthy intact skin margin. All excisions were performed to include the subcutaneous tissue in the specimen. Then, an adequate amount of skin was outlined on the preauricular region or on the neck by using a template. There is a surprising width of skin between the tragus and the sideburns, particularly when stretched. Skin graft was harvested in such a way that it included the superficial layers of subcutaneous tissue. The graft donor site in the neck or preauricular site was primarily closed. The thickness of so-called composite-skin graft was adjusted according to the recipient site requirements. After a meticulous hemostasis in the recipient bed, the defect was covered by the composite-skin graft. Interrupted $7 / 0$ nonabsorbable stitches were used to suture the graft in place. Figures 1 and 2 illustrate the use of the technique for closure of defects on the left alar region and nasal tip and dorsum, respectively. A tie-over dressing was always applied to avoid seroma or hematoma formation and to ensure immobilization of the graft and maximal graft take.

\section{Patients}

The technique of composite-skin grafting was used for defects remaining after the excision of basal cell carcinomas in a series of 15 patients ( 4 males, mean age: 61 years): lateral nasal region ( 5 cases), nasal tip (4 cases), dorsum ( 3 cases), alar lobule ( 2 cases), and soft triangle (1 case). The size of the composite-skin graft for coverage varied between $4 \times 3.5 \mathrm{~cm}$ (maximum) and $1 \times 1 \mathrm{~cm}$ (minimum) (Table 1$)$. The mean follow-up period was 14.2 months. In the early postoperative period, in one case there was a superficial epidermolysis on the composite-skin graft which 

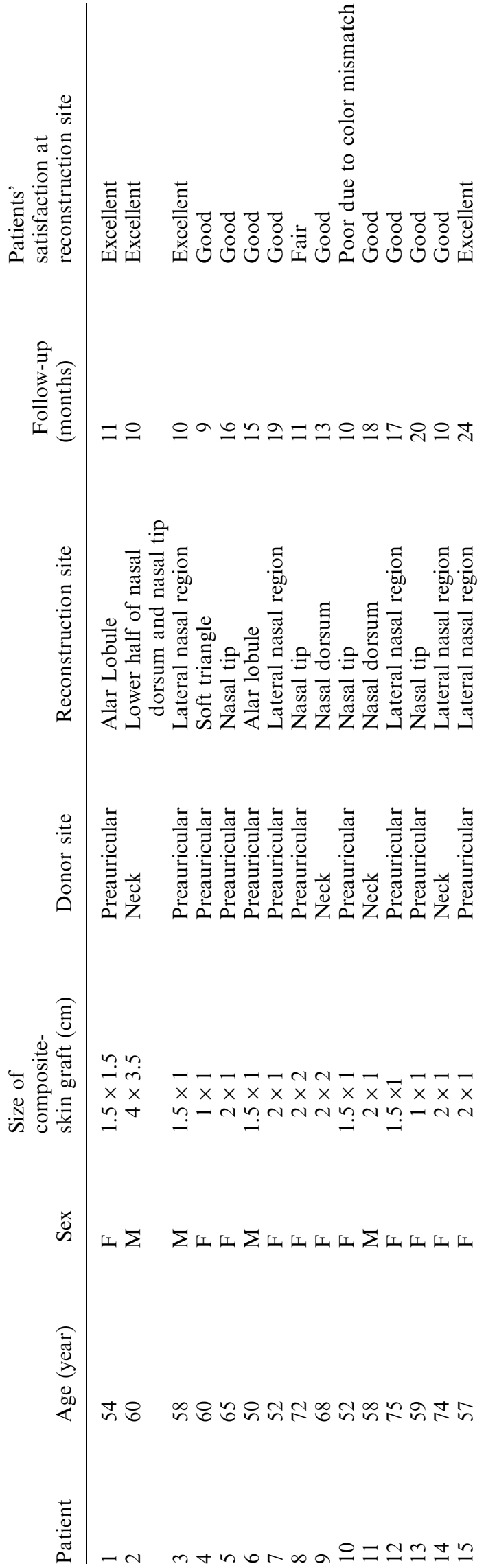

multiple incisions on the leading surface of the nose makes the procedure less attractive [4]. Besides, dogears requiring secondary operations are not infrequent. Moreover, a trap-door phenomenon could result from scar contracture with an unpleasant aesthetic appearance. Banner flap utilized for closure of nasal tip defects may lead to asymmetrical elevation of the alar rims or alar notching, which tends to occur as the donor area of the flap is closed [5]. Larger tip defects can be reconstructed with dorsal nasal flaps [6], axial frontonasal flap [7] or V-Y advancement island flaps [8,9], however, their use is technically demanding and requires experienced planning to avoid untoward outcomes. Reconstruction of alar region poses a significant difficulty for the surgeon because local flap options are limited and utilized techniques for this purpose do not yield better aesthetic results compared to that of skin grafting.

Among a variety of treatment methods listed previously, we favor primary surgical excision of basal cell carcinomas with immediate or delayed closure followed by composite-skin graft harvested from preauricular site or the neck.

Although the same principles with regard to revascularization and harvesting are applicable, the technique of composite-skin grafting has several advantages over the conventional full-thickness skin grafts and local flaps. While full-thickness skin grafts remain inadequate regarding thickness, compositeskin graft provides a relatively thicker tissue and alleviates occurrence of associated contour deformities. Because composite-skin grafts possess superficial layers of subcutaneous fat tissue, this increases the thickness of the graft. This results in a better match in thickness in the reconstruction site, especially in patients who have a thick glabrous skin on the nose. Although the color and texture match was not as good as that provided with local flaps raised from adjacent skin, composite-skin graft was superior to the conventional full-thickness skin grafts, especially when preauricular site was used as donor.

The preauricular region has previously been described as an ideal donor site to the head and neck. Preauricular skin is probably the most accessible of donor sites on the head and neck and this allows a graft of the appropriate size and shape in most cases [10-12].

In our experience, preauricular donor site is also far superior to other available skin grafting techniques for the nose. Although retroauricular sulcus will yield a rather large piece of tissue with a hidden scar, we have found these to be a poor match in color, thickness, and texture. It is also a difficult area to reach both for harvesting the graft and closing the defect. The supraclavicular fossa gives a better color match, but the thickness and texture are not optimal. Supraclavicular scars are also unsightly, especially for women. The preauricular incision heals very nicely, particularly in the older age groups in which cutaneous neoplasm is most likely to occur (Fig. 1F). Nevertheless, in some male patients, the neck, su- 

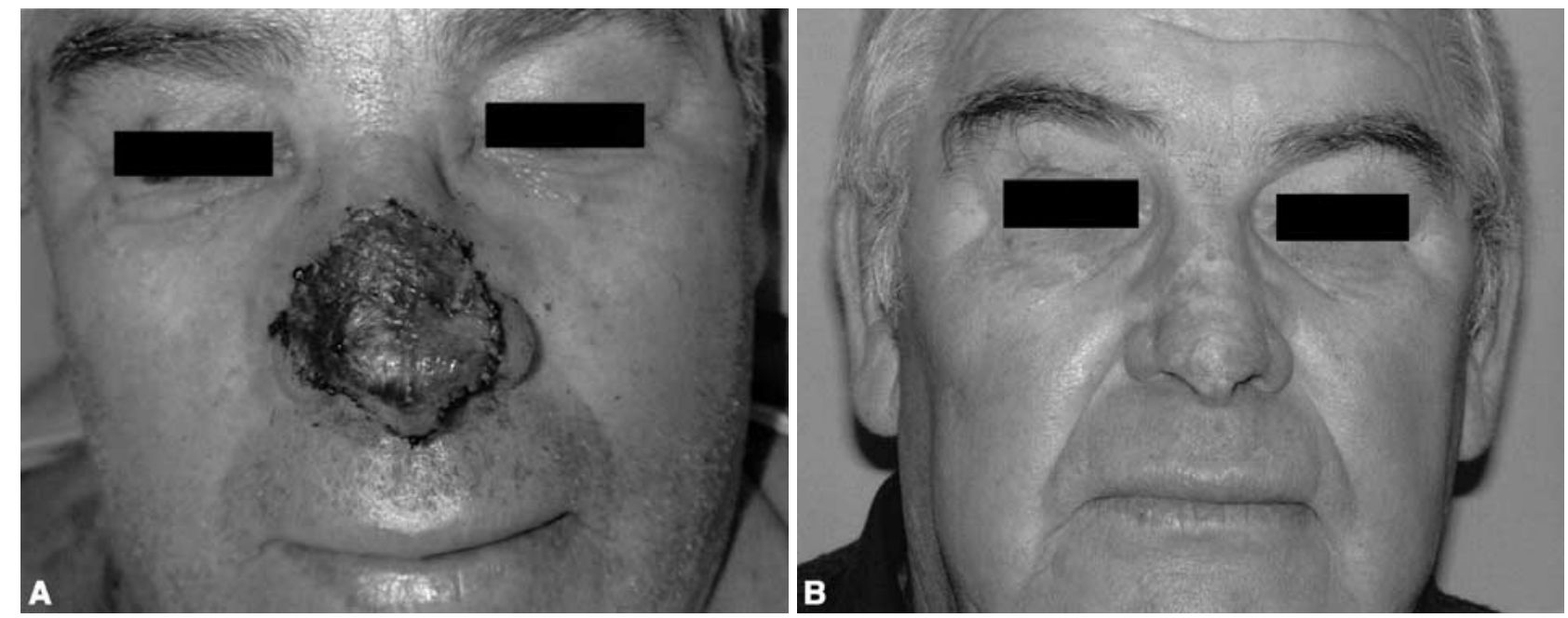

Fig. 3. (A) Figure shows the development of epidermolysis on the composite-skin graft in patient 2 one week after the surgery, (B) Postoperative frontal view at 10-month follow-up demonstrates well-adapted composite-skin graft on the nose with satisfactory aesthetic appearance.
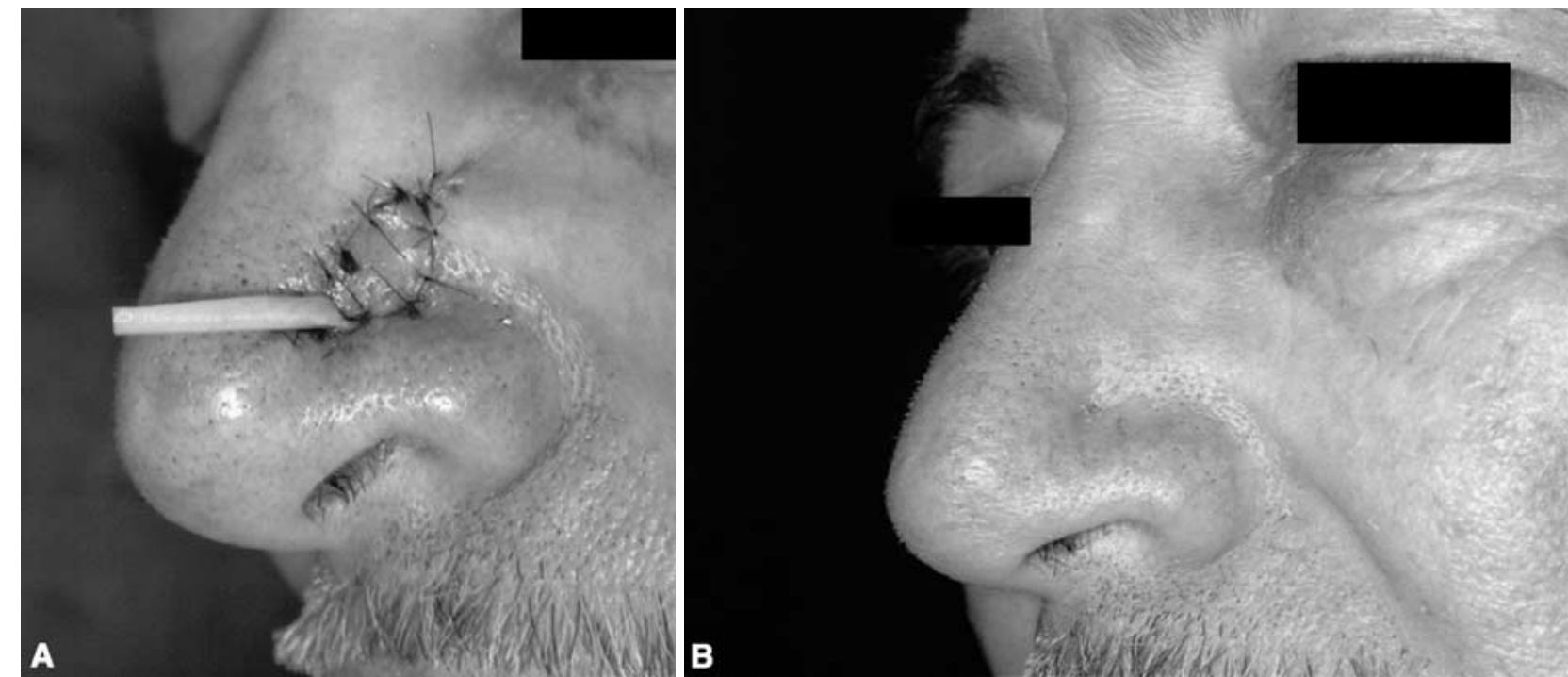

A

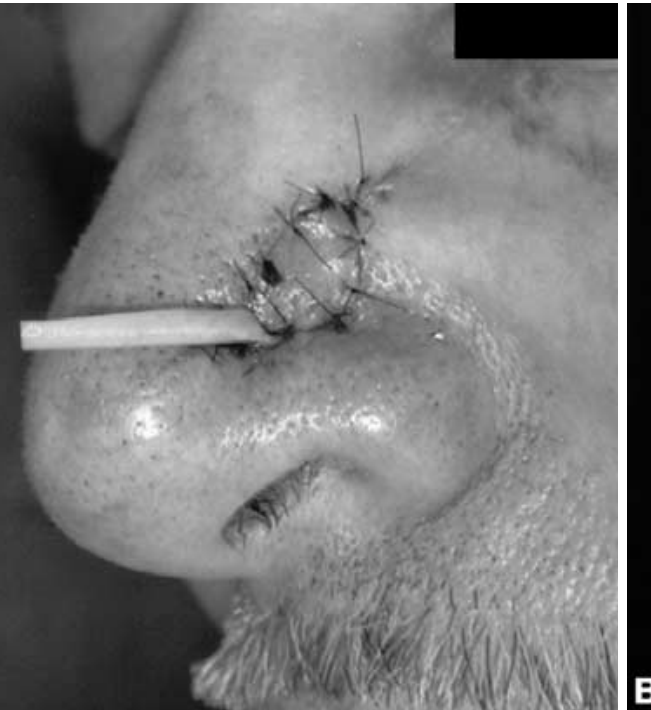

Fig. 4. Patient 3: (A) Close-up view showing closure of a defect on the lateral aspect of nose with a composite-skin graft harvested from preauricular region. (B) Postoperative picture at 10 month follow-up, showing no visible scar.

praclavicular or retroauricular areas, should be preferred to avoid unwanted hair growth on the transplanted skin, which could be occasionally problematic with large preauricular grafts.

Another significant advantage of the compositeskin grafting is that it is technically less demanding compared to the local flap and prominent extra scars are avoided. With this technique scars are well concealed both in the reconstruction site and in the skin graft donor region. Utilization of a fine surgical technique and fine sutures (7/0 prolene), early stitch removal and the application of the technique in fairskinned individuals, known to improve the outcome, are also beneficial for obtaining an optimal aesthetic appearance. The pleasing aesthetic result in all cases of this series was demonstrated by the blending in with the surrounding tissue and the absence of the noticeable depression at the grafted site (Figs. 3B, 4B, $5 \mathrm{C})$. It is recommended to extend the excision margin of the basal cell carcinoma up to the limits of the aesthetic unit, if possible, to achieve en-bloc coverage of the unit (Fig. 6). If the nasal defect encompasses more than $50 \%$ of the aesthetic unit, it can be enlarged to the boundaries of the aesthetic unit during the reconstruction. On the other hand, if the skin defect is less than $50 \%$ of the aesthetic unit, it is best to ignore the aesthetic unit rather than convert the defect into a larger loss [13-15].

Of course, there are some concerns that should be addressed in the technique of composite-skin graft- 

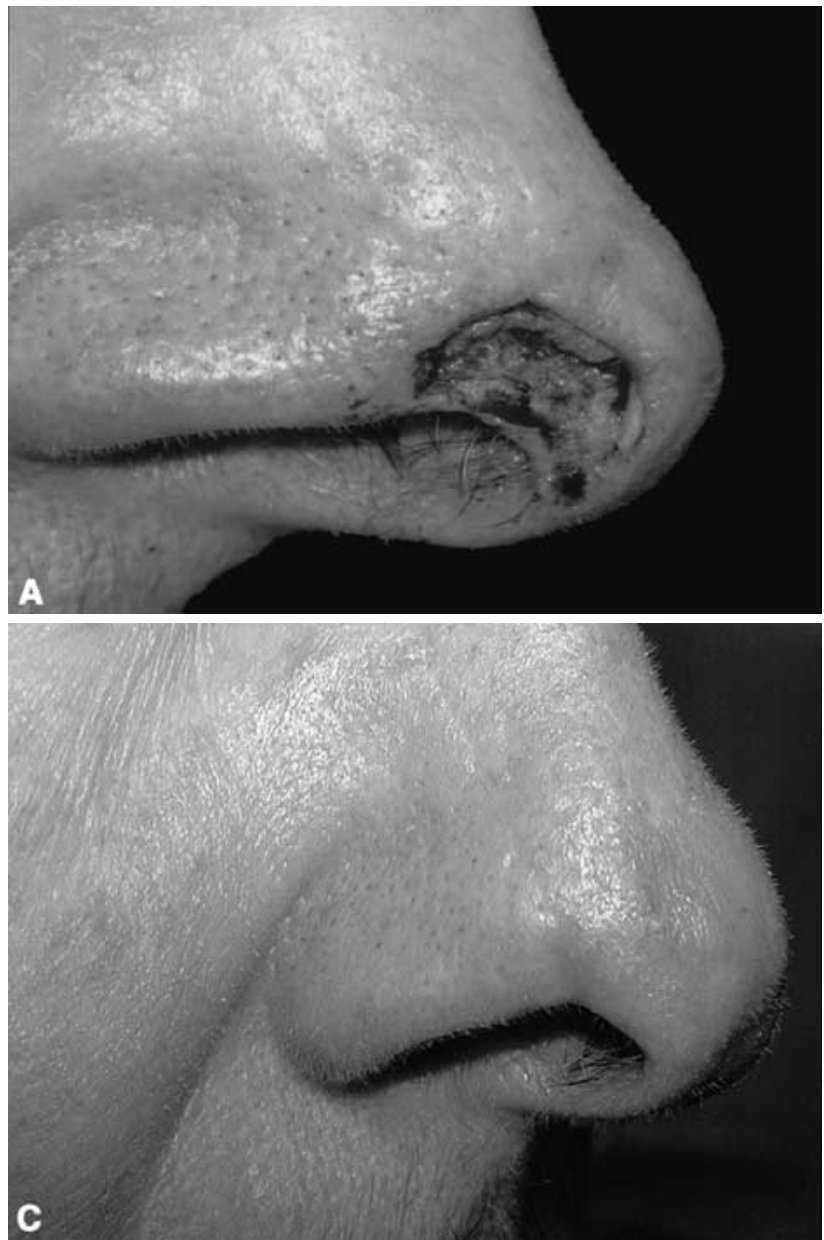

ing. In dark-skinned individuals color mismatch can be problematic. The size of the composite graft with regard to revascularization from the recipient bed is limited. The largest composite skin graft used in this series was $4 \times 3.5 \mathrm{~cm}$. Initially epidermolysis developed resulting in superficial sloughing of the epidermis. This, however, eventually was replaced with a new layer of epidermis without the need for surgical treatment. Another concern of course is to have a well-vascularized recipient bed, a prerequisite to ensure graft take. Thus, defects with bare bone or cartilage are conceivably not suitable for coverage with composite-skin grafts.

\section{Conclusions}

Composite-skin grafting for reconstruction of basal cell carcinoma defects on the nose was successfully used in a series of 15 patients. The preauricular site of the face and the neck provided an excellent color and texture match. The technique offers several advantages over local flap and conventional full-thickness skin grafting techniques. Composite-skin grafting provides an adequate amount of skin thickness that is comparable to skin flaps, and contour deformities

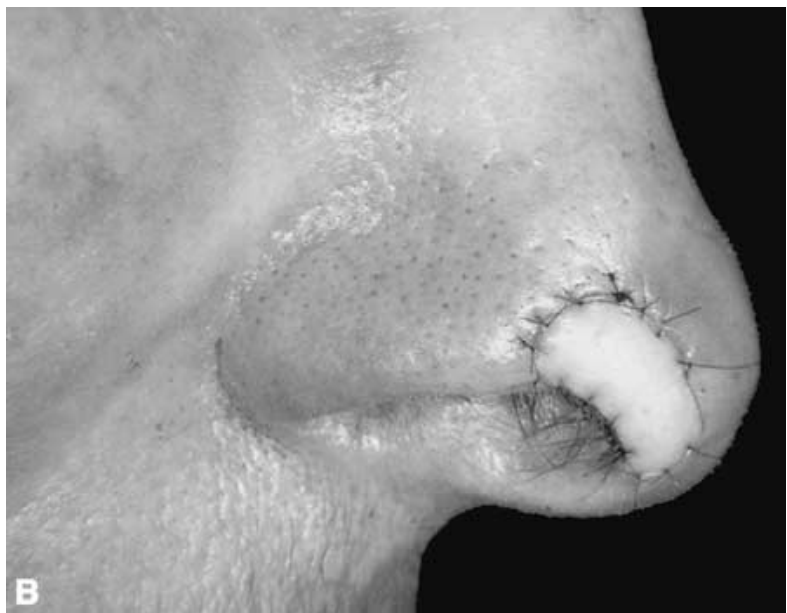

Fig. 5. Patient 4: (A) Close-up view of a defect in the right soft triangle after basal cell carcinoma excision, (B) closure with preauricular composite-skin graft, and (C) late postoperative picture at nine-month follow-up demonstrates aesthetically pleasing closure.

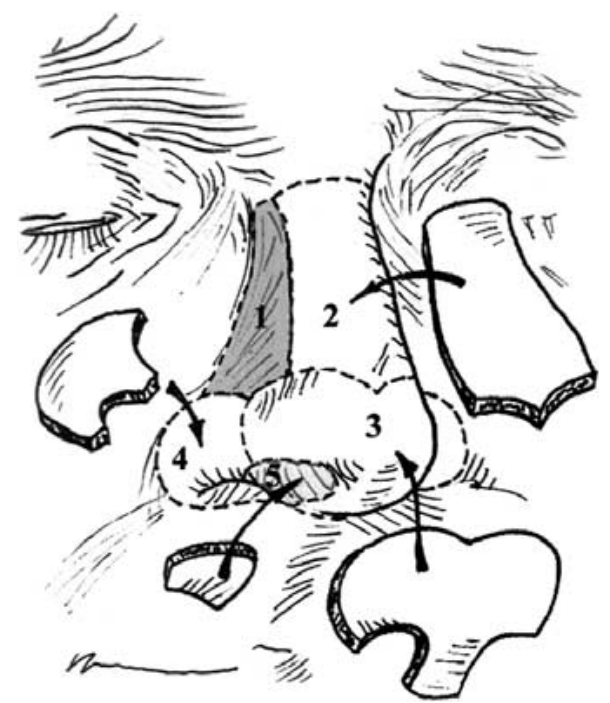

Fig. 6. Aesthetic units of the nose. 1: Lateral nasal region, 2: dorsum, 3: tip, 4: alar lobule, and 5: soft triangle.

associated with full-thickness skin grafts are avoided. Composite-skin grafting procedure is simpler, practical, and technically less demanding compared to local flaps. Furthermore, extra scars, dog-ears, trap- 
door phenomenon, obliteration of facial definition are avoided. The procedure avoids long suture lines running out of the cosmetic subunit and distortion of the nose does not occur. Based on the aesthetically satisfactory results of our cases, we favor the use of this technique as an alternative to other techniques for covering nasal defects after basal cell carcinoma excisions in selected individuals.

\section{References}

1. Stal S, Spira M: Basal and squamous cell carcinoma of the skin. In: Grabb SJ, Beasley RW, Thorne CHM (Eds.) Plastic surgery. Lippincott-Raven Publishers, New York, pp 170-120, 1997

2. Fitzpatrick TB: The validity and practicality of sun reactive types I through VI. Arch Dermatol 124:869, 1988

3. Preston DS, Stern RS: Nomelanoma cancers of skin. $N$ Engl J Med 327:1649, 1992

4. Matarasso A: Bilobed nasal skin flaps. In: Strauch B, Vasconez LO, Hall-Findlay EJ (Eds.) Grabb's Encyclopedia of Flaps: Head and Neck, Vol 1. Little Brown, Boston, pp 135-137, 1990

5. Mendelson BC, Masson JK: Banner-shaped nasal skin flap. In: Strauch B, Vasconez LO, Hall-Findlay EJ
(Eds.) Grabb's Encyclopedia of Flaps: Head and Neck, Vol 1. Little Brown, Boston, pp 141-145, 1990

6. Rieger RA: A local flap for repair of the nasal tip. Plast Reconstr Surg 40:147, 1967

7. Marchac D, Toth B: The axial frontonasal flap revisited. Plast Reconstr Surg 76:686, 1985

8. Doermann A, Hauter D, Zook EG, et al.: V-Y advancement flaps for closure of nasal defects. Plast Reconstr Surg. 84:916, 1989

9. Ohsumi N, Ishikawa T, Shibata Y: Reconstruction of nasal tip defects by dorsonasal V-Y advancement island flap. Ann Plast Surg 40:18, 1998

10. Breach NM: Pre-auricular full-thickness skin grafts. $\mathrm{Br}$ J Plast Surg 31:124, 1978

11. Corwin TR, Klein AW, Habal MB: The aesthetics of the preauricular graft in facial reconstruction. Ann Plast Surg 9:312, 1982

12. Johnson T, Zide MF: Freehand full-thickness grafting for facial defects: a review of methods. J Oral Maxillofac Surg 55(10):1050, 1997

13. Gonzalez-Ulloa M: Restoration of the face covering by means of selected in regional aesthetic units. Br J Plast Surg 9:212, 1956

14. Burget GC: Aesthetic restoration of the nose. Clin Plast Surg 12:463, 1985

15. Burget GC, Menick FJ: The subunit principle in nasal reconstruction. Plast Reconstr Surg 76:239, 1985 